\title{
Classification, physicochemical, soil fertility, and relationship to Coffee robusta yield in soil map unit selected
}

\author{
Posma Marbun $^{1}$ (D), Zulkifli Nasution ${ }^{1}$, Hamidah Hanum¹ ${ }^{1}$, Abubakar Karim² ${ }^{(\mathbb{D}}$
}

\begin{abstract}
${ }^{1}$ Universitas Sumatera Utara, Faculty of Agriculture, Program Study of Agrotechnology, Medan 20155, Indonesia. ${ }^{2}$ Universitas Syiah Kuala, Faculty of Agriculture, Program Study of Agrotechnology, Banda Aceh 23111, Indonesia. Contact authors: posmamarbun12@gmail.com, zulnasution@usu.ac.id, hamidah.azhar@yahoo.co.id, karim.abubakar@unsyiah.ac.id Received in September 9, 2020 and approved in October 26, 2020
\end{abstract}

\section{ABSTRACT}

The research was aimed to classify, characterize the physicochemical properties, determine the fertility of the soil, and to obtain the relationship of soil fertility on the character yield for Coffee robusta in the 10 units of the soil map (SMUs) selected. This research was conducted in Silima Pungga-Pungga sub-District, Dairi District, North Sumatra Province, Indonesia from July 2014 to June 2017. This research was conducted by overlay the maps, classifying soil profiles, characterizing soil, soil fertility assessing, and regression analysis of soil fertility with the yield for Coffee robusta using IBM SPSS Statistics v.20 software. The result showed the ten from 18 SMUs selected for Coffee robusta had the highest area in sequentially, namely SMU 11,14 , and 1. Based on the ten SMUs selected, found in two representative soil profiles, include the profile 1 (SMU 1, 2, 8, 9, 11, 13, 14, 16, 18) covering an area of $1,703.30$ ha with the inceptisol and profile 10 (SMU 10) covering an area of 176.81 ha with the entisol. Inceptisol has greater thesoil physicochemical properties compared to entisol from ten SMUs selected for Coffee robusta. The effect of cation exchange capacity, base saturation, P-total, K-total, and C-organic have significantly increased the productivity of Coffee robusta by $89.30 \%$. However, the effect was not significant to the 100 grains of dry weight.

Key words: Entisol; Coffee robusta; Inceptisol; Overlay; Soil fertility.

\section{INTRODUCTION}

Coffee is a plantation crop that can be supporting the human economy in Indonesia. Statistics of Indonesia (2019) reported that Indonesia coffee export volume over the last 10 years (2010 until 2019) experienced fluctuating and reached $355,766.5$ ton with total area of 1,258 thousand ha in year 2019. Statistics of Indonesia (2019) also reported the countries that were the main export destinations for Indonesia coffee found in the United States by 58,666.2 ton, and were followed in Italy of 35,452.2 ton, Malaysia of 34,662.2 ton, Egypt of 34,285 ton, and Japan of 25,587.8 ton. It is shows that coffee yield has the opportunity to be exported and can support the income of Indonesia coffee farmers.

The coffee area is dominated by smallholder with an area of $1,194,081$ ha $(96.16 \%)$ of the total coffee area in Indonesia, while the residual of 47,632 ha $(3.84 \%)$ are government and private estate. Farmers in Indonesia grow three types of coffee, such as robusta, arabica, and liberica. Coffee robusta plant is the most widely grown by farmers in Indonesia, followed by arabica and liberica with area reached 879,117 ha $(70.80 \%)$ and 314,963 ha $(25.36 \%)$, respectively. The distribution of the planting areas for Coffee robusta in sequentially found in the regions Sumatra of 596,610 ha, Java of 106,161 ha, Nusa Tenggara and Bali of 88,108 ha, Sulawesi of 57,427 ha, Kalimantan of 26,315 ha, Maluku and Papua of 4,495 ha (Indonesian Agency for Agricultural Researchand Development, 2015).

Statistics of Indonesia (2019) reported that North Sumatra Province has a coffee area of 97.50 thousand ha from the Sumatra region and is in $4^{\text {th }}$ place after South Sumatra Province of 251 thousand ha, Lampung of 156.90 thousand ha, Aceh of 125.30 thousand ha. Statistics of Sumatera Utara (2018) reported that the area for Coffee robusta planted in North Sumatra was 17,437.64 ha with the yield up to $6,788.70$ ton and the largest area for planting in Dairi District was 8,427 ha or $48.33 \%$ compared to other districts in 2018. Thus the productivity for Coffee robusta in Dairi District was $402.02 \mathrm{~kg} \mathrm{ha}^{-1}$ and was classified as lower compared to the national rate of $723.01 \mathrm{~kg} \mathrm{ha}^{-1}$.

The low productivity Coffee robusta in Dairi District could be caused by several factors, one of them is the decrease in land productivity in supporting coffee yield. Thus it is necessary to research the status of soil fertility at Dairi District in deta il, covering the overlay Soil Map Unit (SMU), the classification and characterization of soil, and analysis of the relationship between soil fertility on the yield characteristics for Coffee robusta. It has been reported that the classification and char acterization of soil in several plantation areas for Coffee robusta in Dairi District. Marbun et al. (2016) reported that an area of 891.99 ha in the SMU 3 until 7 in the Silima Pungga- P u ngga sub-District, Dairi District had inceptisol orders with the character the cation exchange capacity (CEC) was classified as low until very high, base saturation (BS) and C-organic were classified as very low until low. Marbun et al. (2018) also added a land area of 2,241.42 ha at SMU 12, 15, and 17 from Silima Pungga-Pungga sub-District, Dairi District had andisol order with the characters CEC was classified as low until moderate, $\mathrm{BS}$ and $\mathrm{C}$-organic were classified as very low until low. 
The reports the previous research is incomplete because there are still areas cultivation for Coffee robusta of 1880.11 ha or 10 SMUsin the Silima Pungga-Pungga sub-District, Dairi Distric $t$ that has not been classified in detail based overlay mapping of land. The research was aimed to (1) classify, charact erize the soil physicochemical properties, determine the status of soil fertility, and (2) obtain the relationship CEC, BS, P-total, K-total, and C-organic toward productivity and weight of 100 grains of dry weight for Coffee robusta at the ten SMUs selected in Silima Pungga-Pungga sub-District, Dairi District, North Sumatra Province, Indonesia.

\section{MATERIAL AND METHODS}

\subsection{Research Area for Coffee robusta}

The res earch was conducted in the Silima PunggaPungga sub-District, Dairi District, North Sumatra Province, Indones ia with the coordinates $2^{\circ} 80^{\prime}-2^{\circ} 88^{\prime}$ NL and $98^{\circ} 04^{\prime}-$ $98^{\circ} 17^{\prime} \mathrm{EL}$ in the altitude of 400 until $800 \mathrm{~m}$ above sea level from July 2014 until June 2017. Soil analysis was conducted at the Research and Technology Laboratory, Faculty of Agriculture, Universitas Sumatera Utara, Medan, Indonesia.

\subsection{Map Overlay and Soil Profile Classification}

A survey was conducted to make a soil profile. Soil profiles were conducted on Soil Map Unit (SMU) based on a map overlay technique between soil type, altitude, and slope with a scale of 1 : 25,000 respectively using ArcView GIS 3.2a (Figure 1).

Observa tion of the morphology and characteristics of the soil in each profile was conducted using the reference book such as "Guidelines of Soil Observation in Field" by IAARD p ress and "Key to Soil Taxonomy 2014" by Soil Survey Staff USDA. Soil profile classification was conducted by determining the epipedon horizon, subsurface horizon, and other identifiers properties, then it was determined the order, sub-order, great group, and sub-group.
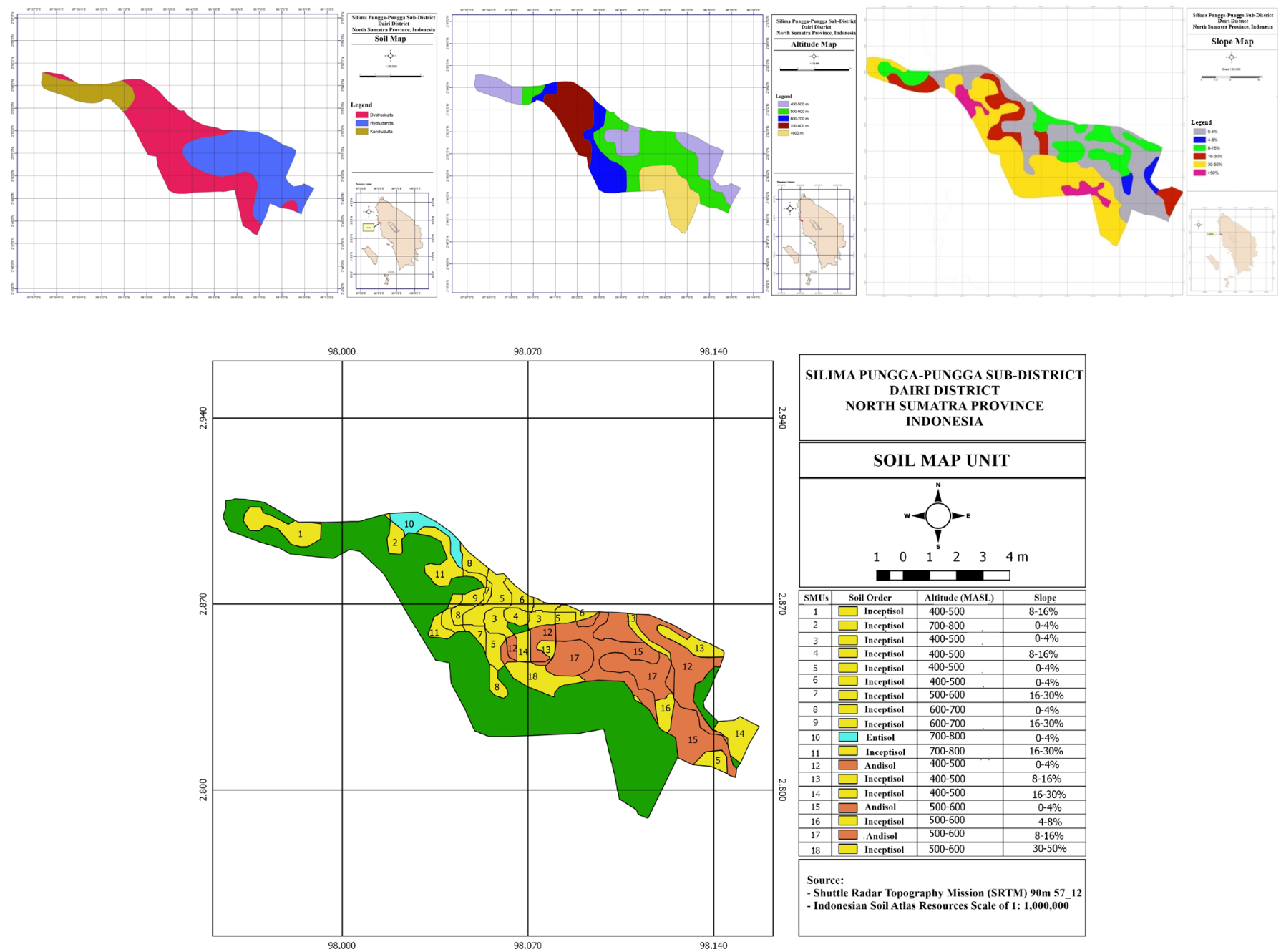

Figure 1: SMUs for Coffee robusta with an overlay technique between maps of soil types, altitude, and slope in Silima PunggaPungga Sub-District, Dairi District, North Sumatra Province, Indonesia. 


\subsection{Soil Physicochemical Properties}

Soil ph ysicochemical properties were conducted by taki ng soil samples from each horizon at 10 SMUs selected, include Cation Exchange Capacity (CEC) and Base Saturat ion (BS) with the extraction method of $\mathrm{NH}_{4} \mathrm{OAc}$ $(\mathrm{pH}$ 7), C-organic with the Walkley \& Black method, soil $\mathrm{pH}\left(\mathrm{H}_{2} \mathrm{O}, \mathrm{KCl}, \mathrm{NaF}\right)$, salinity with the platinum electrode method, soil color using the Munsell Soil Color Chart book, soil structure, soil consistency with the Atterberg method, soil texture with the Hydrometer method, and bulk density using a ring sample.

\subsection{Soil Fertility Assessment}

Assessm ent of soil fertility was determined by the mai $n$ soil chemical characteristics, such as: CEC and BS, meanwhile other soil chemical characteristics are det ermined through P-total, K-total, and C-organic. Overall, soil chemical characteristics were obtained using a parametric method, and the soil fertility assessment was determined based on the CEC, BS, $\mathrm{P}_{2} \mathrm{O}_{5}, \mathrm{~K}_{2} \mathrm{O}$, and C-organic on upland acid soils in Southeast Asia by Dierolf, Fairhurst and Mutert (2001).

\subsection{Multiple Linear Regression Analysis}

The productivity and 100 grains of dry weight for Coffee robusta were collected from 30 farmers in each SMU selected and then weighed. Multiple linear regression analysis (F-test and determination coefficient) was performed in the influence of CEC, BS, P-total, K-total, and C-organic on productivity and 100 grains of dry weight for Coffee robusta using IBM SPSS Statistics v.20 software.

\section{RESULTS}

\subsection{Soil Map Unit (SMU)Selected}

The ten of 18 SMUs were selected for Coffee robusta in Silima Pungga-Pungga sub-District, Dairi District, North Sumatra Province (Table 1).

Based on the overlay results obtained the ten of 18 SMUs selected for Coffee robusta with three SMUs have the highest an area in sequentially,namely SMU 11 (great group dystrudept, altitude ranged by 700 to $800 \mathrm{~m}$, slope ranged by 16 to $30 \%$ ), SMU 14 (great group dystrudept, altitude ranged of 400 to $500 \mathrm{~m}$, slope ranged of 16 to $30 \%$ ) and SMU 1 (great group dystrudept, altitude ranged by 400 to $500 \mathrm{~m}$, slope ranged by 8 to $16 \%$ ).

\subsection{Soil Classification for Coffee robusta}

Determination of the epipedon horizon, subsurface horizon, other identifiers properties, the order, sub-order, great group, sub-group of $10 \mathrm{SMUs}$ selected for Coffee robusta in Silima Pungga-Pungga sub-District, Dairi District (Tables 2 and 3). Based on ten SMUs selected, obtained two representative soil profiles in Silima Pungga-Pungga subDistrict, Dairi District, such as profile 1 include SMU 1, 2, 8, $9,11,13,14,16,18$ covering an area of $1,703.30$ ha with the umbric epipedon, the cambic subsurface horizon, inceptisol order, udept sub-order, dystrudept great group, typic dystrudept sub-group, and it had the Ap-B-C horizon. The profile 10, only SMU 10 covering an area of 176.81 ha with the ochric epipedon, it does not have a characteristic subsurface horizon, entisol order, orthent sub-order, udorthent great group, typic udorthent sub-group, and it had the horizon Ap-A-C.

Table 1: The soil map unit (SMU) was selected for Coffee robusta in Silima Pungga-Pungga sub-District, Dairi District, North Sumatra Province, Indonesia.

\begin{tabular}{|c|c|c|c|c|c|}
\hline SMUs & Great Group & Altitude (m) & Slope $(\%)$ & Villages & Area (ha) \\
\hline & $\begin{array}{l}\text { SMU } 1 \\
\text { Dystrudept } \\
400-500\end{array}$ & & $8-16$ & Lae Rambong & 253.16 \\
\hline SMU 2 & Dystrudept & $700-800$ & $0-4$ & Lae Ambat & 22.22 \\
\hline SMU 8 & Dystrudept & $600-700$ & $0-4$ & Lae Ambat & 184.99 \\
\hline SMU 9 & Dystrudept & $600-700$ & $16-30$ & Lae Ambat & 133.68 \\
\hline SMU 10 & Udorthent & $700-800$ & $0-4$ & Lae Ambat & 176.81 \\
\hline SMU 11 & Dystrudept & $700-800$ & $16-30$ & Lae Ambat & 293.24 \\
\hline SMU 13 & Dystrudept & $400-500$ & $8-16$ & Sinatah, Parongil, Polding Anak-anak, Sumbari & 247.95 \\
\hline SMU 14 & Dystrudept & $400-500$ & $16-30$ & Palipi & 261.02 \\
\hline SMU 16 & Dystrudept & $500-600$ & $4-8$ & Bakal Gajah, Sumbari, Uruk & 89.88 \\
\hline SMU 18 & Dystrudept & $500-600$ & $30-50$ & $\begin{array}{l}\text { Parongil, Longkotan, Palipi, Tungtung Batu, Polding Anak-anak, } \\
\text { Longkotan, Tungtung Batu, Siboras, Uruk Belin, Bakal Gajah, } \\
\text { Sumbari, Lae Panginuman, Sumbari, Bonian }\end{array}$ & 217.16 \\
\hline Total & & & & & $1,880.11$ \\
\hline
\end{tabular}




\subsection{Soil Physicochemical Properties for Coffee robusta}

The soil physicochemical properties in each representative soil profile of ten SMUs selected for Coffee robusta in Silima Pungga-Pungga sub-District, Dairi District could be seen in Tables 4 and 5. Based on the physical properties of ten SMUs selected, showed that the profile 1 (SMU 1, 2, 8, 9, 11, 13, 14, 16, 18) had the soil structure of the granular until blocky, the soil consistency of the soft until slightly hard, the soil texture of the sandy clay loam until clay, and bulk density ranged of 1.04 to $1.12 \mathrm{~g} . \mathrm{cm}^{-3}$. Profile 10 (SMU 10) had the soil structure of the granular, the soil consistency of the soft until very soft, soil texture of sandy loam until sandy clay loam, and bulk density ranged of 1.18 to $1.26 \mathrm{~g} . \mathrm{cm}^{-3}$.

Based on the chemical properties of ten SMUs selected for Coffee robusta in Silima Pungga-Pungga sub-District, Dairi District, it was obtained that profile 1 (SMU 1, 2, 8, 9, $11,13,14,16,18)$ had the soil $\mathrm{pH}$ had strongly acid to acid, CEC ranged of13.21 until $21.09 \mathrm{me} / 100 \mathrm{~g}$ (low to moderate), C-organic ranged of 0.49 until $1.70 \%$ (very low until low), BS rangedof 15.13 until $18.51 \%$ (very low), Ca-exchangeable was classified as very low, K-exchangeable was classified as low, Na-exchangeable was classified as low until moderate, and Mg-exchangeable was classified as low until high. In profile 10 (SMU 10) had the soil $\mathrm{pH}$ was classified as strongly acid to acid, CEC ranged of 9.99 until 11.89 me/100 g (low), C-organic ranged by 0.48 until $1.36 \%$ (very low until low), BS ranged of 14.43 until $17.00 \%$ (very low), Ca- and $\mathrm{Mg}$-exchangeable were classified as very low, K-exchangeable was classified as very low to low, and Na-exchangeable was classified as low. The salinity rate in both soil profiles were classified as very low.

\subsection{Soil Fertility Assessment}

The soil fertility rate of inceptisol and entisol of ten SMUs selected for Coffee robusta in Silima Pungga-Pungga sub-District, Dairi District (Table 6). The result showed that the inceptisol at SMU 1, 8, 9, 11, and 16 had the soil fertility were classified as very low, meanwhile at SMU 2, 13, 14, and 18 were classified as low. The entisol order (SMU 10) had the soil fertility was classified as very low.

\subsection{Multiple Linear Regression Analysis between Soil Fertility to Yield Characters for Coffee robusta}

The effect of CEC, BS, P-total, K-total, and C-organic on the productivity and 100 grains of dry weight for Coffee robusta in Silima Pungga-Pungga sub-District, Dairi District (Table 7). The result showed that the effect CEC, BS, P-total, K-total, and C-organic were significantly affected by the productivity Coffee robusta with a value of 0.8930 . It was indicated that CEC, BS, P-total, K-total, C-organic could be increasing the productivity Coffee robusta by 89.30\%. The CEC, BS, P-total, K-total, and C-organic was not significant effect on the 100 grains of dry weight for Coffee robusta. However, it had a value of 0.7640 . It was indicated that CEC, BS, P-total, K-total, C-organic could be increasing the 100 grains of dry weight for Coffee robusta by $76.40 \%$.

Table 2: Establishment of the epipedon horizon, subsurface horizon, and other identifiers properties in 10 SMUs selected for Coffee robusta in Silima Pungga-Pungga sub-District, Dairi District, North Sumatra Province, Indonesia.

\begin{tabular}{|c|c|c|c|c|}
\hline Soil Profiles & SMUs & Epipedon horizon & Subsurface horizon & Other identifiers properties \\
\hline 1 & $\begin{array}{l}1 ; 2 ; 8 \\
9 ; 11 \\
13 ; 14 \\
16 ; 18\end{array}$ & $\begin{array}{l}\text { Including the umbric; because it is } \\
\text { located above the soil surface; with } \\
\text { color value and chroma of } 3 \text { or less } \\
\text { (humid); base saturation }<50 \% \text { and } \\
\text { the soil is humid for more than } 3 \\
\text { months }\end{array}$ & $\begin{array}{l}\text { Including the cambic; because it had } \\
\text { the sandy loam texture; the thickness } \\
\text { of the horizon is more than } 15 \mathrm{~cm} \text {; } \\
\text { the absence of clay illuviation process } \\
\text { and not part of the Ap horizon and } \\
\text { does not experience aquic conditions }\end{array}$ & $\begin{array}{l}\text { Has the udic humidity regime because } \\
\text { the soil has never been dry in } 90 \\
\text { days (cumulative); which is more } \\
\text { than } 90 \text { days or the average rainfall } \\
\text { data for wet months ranged by }\end{array}$ \\
\hline 10 & 10 & $\begin{array}{l}\text { Including the ochric; because the } \\
\text { value or chroma is more than } 3 \\
\text { (humid). }\end{array}$ & $\begin{array}{l}\text { It does not have a characteristic } \\
\text { subsurface horizon because it is not } \\
\text { yet developed }\end{array}$ & $\begin{array}{l}300 \text { days (cumulative); and has the } \\
\text { isohipertermic temperature soil regime }\end{array}$ \\
\hline
\end{tabular}

Table 3: Establishment of order, sub-order, great group, and sub-groupof 10 SMUs selected for Coffee robusta in Silima PunggaPungga sub-District, Dairi District, North Sumatra Province, Indonesia.

\begin{tabular}{|c|c|c|c|c|c|}
\hline Soil Profiles & SMUs & Order & Sub-order & Great group & Sub-group \\
\hline 1 & $\begin{array}{l}1 ; 2 ; 8 \\
9 ; 11 ; \\
13 ; 14 \\
16 ; 18\end{array}$ & $\begin{array}{l}\text { Including the inceptisol; because it had } \\
\text { an umbric epipedon and had subsurface } \\
\text { horizon of cambic at a depth of less than } \\
100 \mathrm{~cm} \text { from the ground surface }\end{array}$ & $\begin{array}{l}\text { Including the udept } \\
\text { sub-order because it } \\
\text { has udic soil moisture } \\
\text { regime }\end{array}$ & $\begin{array}{l}\text { Including the } \\
\text { dystrudept because } \\
\text { it has another udept }\end{array}$ & $\begin{array}{l}\text { Including the typic } \\
\text { dystrudept sub-group } \\
\text { because it has others } \\
\text { dystrudept }\end{array}$ \\
\hline 10 & 10 & $\begin{array}{l}\text { Including the entisol; because it does } \\
\text { not have a subsurface horizon and has a } \\
\text { little horizon arrangement }\end{array}$ & $\begin{array}{l}\text { Including the orthent } \\
\text { sub-order because } \\
\text { it has other entisol } \\
\text { characteristics }\end{array}$ & $\begin{array}{l}\text { Including the } \\
\text { udorthent because } \\
\text { it has other orthent } \\
\text { characteristics }\end{array}$ & $\begin{array}{l}\text { Including the typic } \\
\text { udorthent sub-group } \\
\text { because it has others } \\
\text { udorthent }\end{array}$ \\
\hline
\end{tabular}


Table 4: Physical characteristics of soil profiles from 10 SMUs selected in Coffee robusta in Silima Pungga-Pungga sub-District, Dairi District, North Sumatra Province, Indonesia.

\begin{tabular}{|c|c|c|c|c|c|c|c|c|c|c|}
\hline \multirow{2}{*}{ Soil Profiles } & \multirow{2}{*}{ Horizons } & \multirow{2}{*}{ Depth (cm) } & \multirow{2}{*}{ Soil Color } & \multirow{2}{*}{ Soil Structure } & \multirow{2}{*}{ Soil Consistency } & \multicolumn{3}{|c|}{ Texture Fraction (\%) } & \multirow{2}{*}{$\begin{array}{c}\text { Soil } \\
\text { Texture }\end{array}$} & \multirow{2}{*}{$\begin{array}{c}\mathrm{BD} \\
\left(\mathrm{g} \cdot \mathrm{cm}^{-3}\right)\end{array}$} \\
\hline & & & & & & Sand & Silt & Clay & & \\
\hline \multirow{3}{*}{$\begin{array}{c}\text { Profile } 1 \\
\text { (SMUs 1; 2; } 8 \\
\text { 9; 11; 13; } 14 ; \\
16 ; 18 \text { ) }\end{array}$} & Ap & $0-34$ & $5 \mathrm{YR} 3 / 3$ & Granular & Soft & 49.84 & 26.56 & 23.60 & SCL & 1.09 \\
\hline & $\mathrm{B}$ & $34-104$ & 5 YR 6/3 & Blocky & Slightly hard & 39.84 & 18.56 & 41.60 & $\mathrm{C}$ & 1.12 \\
\hline & $\mathrm{C}$ & $104-150$ & 5 YR 5/8 & Blocky & Slightly hard & 31.12 & 15.28 & 53.60 & $\mathrm{C}$ & 1.04 \\
\hline \multirow{3}{*}{$\begin{array}{l}\text { Profile } 10 \\
\text { (SMU 10) }\end{array}$} & Ap & $0-29$ & $10 \mathrm{YR} 4 / 6$ & Granular & Soft & 65.12 & 21.28 & 13.60 & SL & 1.18 \\
\hline & A & $29-38$ & $10 \mathrm{YR} 4 / 3$ & Granular & Soft & 59.12 & 21.28 & 19.60 & SL & 1.21 \\
\hline & $\mathrm{C}$ & $38-150$ & $10 \mathrm{YR}$ 5/6 & Granular & Very soft & 53.84 & 19.28 & 26.88 & $\mathrm{SCL}$ & 1.26 \\
\hline
\end{tabular}

Note: Criteria for Soil Texture (SL= sandy loam; $\mathrm{SCL}=$ sandy clay loam; $\mathrm{C}=\mathrm{Clay})$.

Table 5: Chemical characteristics of soil profile from 10 SMUs selected in Coffee robustain Silima Pungga-Pungga sub-District, DairiDistrict, North Sumatra Province, Indonesia.

\begin{tabular}{|c|c|c|c|c|c|c|c|c|c|}
\hline \multirow{2}{*}{ Soil Profiles } & \multirow{2}{*}{ Horizons } & \multirow{2}{*}{ Depth $(\mathrm{cm})$} & \multicolumn{3}{|c|}{ Soil pH } & \multirow{2}{*}{$\begin{array}{c}\text { CEC } \\
(\mathrm{me} / 100 \mathrm{~g})\end{array}$} & \multirow{2}{*}{\multicolumn{2}{|c|}{ C-organic(\%) }} & \multirow{2}{*}{$\begin{array}{c}\mathrm{EC} \\
(\mathrm{dS} / \mathrm{m})\end{array}$} \\
\hline & & & $\mathrm{H}_{2} \mathrm{O}$ & $\mathrm{KCl}$ & $\mathrm{NaF}$ & & & & \\
\hline \multirow{3}{*}{$\begin{array}{c}\text { Profile } 1 \\
\text { (SMUs } 1 ; 2 ; 8 ; 9 ; 11 \\
\text { 13;14;16;18) }\end{array}$} & Ap & $0-34$ & $4.98(\mathrm{~A})$ & 3.65 & 9.82 & $15.21(\mathrm{~L})$ & \multicolumn{2}{|c|}{$1.70(\mathrm{~L})$} & $0.95(\mathrm{VL})$ \\
\hline & B & $34-104$ & $5.06(\mathrm{~A})$ & 3.47 & 9.80 & $13.21(\mathrm{~L})$ & \multicolumn{2}{|c|}{$0.87(\mathrm{VL})$} & $0.55(\mathrm{VL})$ \\
\hline & $\mathrm{C}$ & $104-150$ & $4.36(\mathrm{SoA})$ & 3.67 & 10.30 & $21.09(\mathrm{M})$ & \multicolumn{2}{|c|}{$0.49(\mathrm{VL})$} & $0.55(\mathrm{VL})$ \\
\hline \multirow{3}{*}{$\begin{array}{l}\text { Profile } 10 \\
\text { (SMU 10) }\end{array}$} & Ap & $0-29$ & $4.46(\mathrm{SoA})$ & 4.05 & 9.07 & $9.99(\mathrm{~L})$ & \multicolumn{2}{|c|}{$1.36(\mathrm{~L})$} & $0.40(\mathrm{VL})$ \\
\hline & $\mathrm{A}$ & $29-38$ & $4.61(\mathrm{~A})$ & 4.04 & 9.45 & $8.88(\mathrm{~L})$ & \multicolumn{2}{|c|}{$0.92(\mathrm{VL})$} & $0.75(\mathrm{VL})$ \\
\hline & $\mathrm{C}$ & $38-150$ & $4.75(\mathrm{~A})$ & 4.01 & 9.63 & $11.89(\mathrm{~L})$ & \multicolumn{2}{|c|}{$0.48(\mathrm{VL})$} & $0.70(\mathrm{VL})$ \\
\hline \multirow{2}{*}{ Soil Profiles } & \multirow{2}{*}{ Horizons } & & Exche & angeable $\mathrm{C}$ & $\mathrm{ns}(\mathrm{me} / 1$ & & & & \\
\hline & & Depth (cm) & $\mathrm{Ca}$ & $\mathrm{Mg}$ & $\mathrm{K}$ & $\mathrm{Na}$ & & & \\
\hline Profile 1 & Ap & $0-34$ & $0.926(\mathrm{VL})$ & $0.962(\mathrm{~L})$ & $0.174(\mathrm{~L})$ & $0.240(\mathrm{~L})$ & 5.32 & 5,53 & $15.13(\mathrm{VL})$ \\
\hline (SMUs $1 ; 2 ; 8 ; 9$ & $\mathrm{~B}$ & $34-104$ & $0.999(\mathrm{VL})$ & $0.701(\mathrm{~L})$ & $0.328(\mathrm{~L})$ & $0.528(\mathrm{M})$ & 3.05 & 2.14 & $17.84(\mathrm{VL})$ \\
\hline $11 ; 13 ; 14 ; 16 ; 18)$ & $\mathrm{C}$ & $104-150$ & $1,296(\mathrm{VL})$ & $2.187(\mathrm{H})$ & $0.171(\mathrm{~L})$ & $0.250(\mathrm{~L})$ & 7.58 & 12.79 & $18.51(\mathrm{VL})$ \\
\hline & Ap & $0-29$ & $0.985(\mathrm{VL})$ & $0.344(\mathrm{VL})$ & $0.126(\mathrm{~L})$ & $0.201(\mathrm{~L})$ & 7.82 & 2.73 & $16.58(\mathrm{VL})$ \\
\hline $\begin{array}{l}\text { Profile } 10 \\
\text { (SMU 10) }\end{array}$ & A & $29-38$ & $0.672(\mathrm{VL})$ & $0.176(\mathrm{VL})$ & $0.091(\mathrm{VL})$ & $0.342(\mathrm{~L})$ & 7.38 & 1.93 & $14.43(\mathrm{VL})$ \\
\hline & $\mathrm{C}$ & $38-150$ & $1.486(\mathrm{VL})$ & $0.204(\mathrm{VL})$ & $0.075(\mathrm{VL})$ & $0.256(\mathrm{~L})$ & 19.81 & 2.72 & $17.00(\mathrm{VL})$ \\
\hline
\end{tabular}

Note: Criteria for $\mathrm{pH} \mathrm{H} 2 \mathrm{O}$ (strongly acid/SoA < 4.5; acid/A=4.5-5.5; slightly acid/SiA= 5.5-6.5; neutral/ $\mathrm{N}=6.6-7.5$; slightly alkaline/SAl= 7.6-8.5; alkaline/Al $>8.5$ ); Cation Exchange Capacity/CEC (very low $/ \mathrm{VL}<5 \mathrm{me} / 100 \mathrm{~g}$; low/L= 5-16 me/100 g; moderate/M=17-24 me/100 g; high/H= 25-40 me/100 me/100 g; very high $/ \mathrm{VH}>40 \mathrm{me} / 100 \mathrm{~g}$ ); C-organic (very low $/ \mathrm{VL}<1 \%$; low/L= 1-2\%; moderate/M= 2.01-3\%; high/H=3.01-5\%; very high $/ \mathrm{VH}>5 \%$ ); salinity (very low $/ \mathrm{VL}<1 \mathrm{dS} / \mathrm{m}$; low/L= 1-2 dS/m; moderate/M= 2-3 dS/m; high/H= 3-4 dS/m; very high/VH > $4 \mathrm{dS} / \mathrm{m}$ ) (Soil Research Institute 2009).

Table 6: Soil fertility in Coffee robusta based on 10 SMUs selected in Silima Pungga-Pungga sub-District, Dairi District, North Sumatra Province, Indonesia.

\begin{tabular}{|c|c|c|c|c|c|c|c|c|c|}
\hline Order & Sub-group & SMU & $\begin{array}{c}\text { CEC } \\
\text { (me/100 g) }\end{array}$ & BISA (\%) & P-total (\%) & K-total (\%) & $\begin{array}{c}\text { C-organic } \\
(\%)\end{array}$ & $\begin{array}{l}\text { Productivity } \\
\left(\mathrm{kg} \mathrm{ha}^{-1}\right)\end{array}$ & $\begin{array}{c}\text { Soil } \\
\text { Fertility }\end{array}$ \\
\hline Entisol & $\begin{array}{c}\text { Typic } \\
\text { Udorthent }\end{array}$ & SMU 10 & $10.91(\mathrm{~L})$ & $16.61(\mathrm{VL})$ & $0.04(\mathrm{~L})$ & $0.35(\mathrm{VH})$ & $0.85(\mathrm{VL})$ & 1346.20 & Very low \\
\hline \multirow{4}{*}{ Inceptisol } & \multirow{4}{*}{$\begin{array}{c}\text { Typic } \\
\text { Dystrudept }\end{array}$} & SMU 1 & $14.94(\mathrm{~L})$ & $16.77(\mathrm{VL})$ & $0.03(\mathrm{~L})$ & $0.33(\mathrm{VH})$ & $1.70(\mathrm{~L})$ & 666.05 & Very low \\
\hline & & SMU 2 & $14.31(\mathrm{~L})$ & $28.94(\mathrm{~L})$ & $0.04(\mathrm{~L})$ & $0.33(\mathrm{VH})$ & $1.21(\mathrm{~L})$ & 1082.98 & Low \\
\hline & & SMU 8 & $10.36(\mathrm{~L})$ & $15.98(\mathrm{VL})$ & $0.07(\mathrm{M})$ & $0.29(\mathrm{VH})$ & $1.47(\mathrm{~L})$ & 1363.35 & Very low \\
\hline & & SMU 9 & $10.18(\mathrm{~L})$ & $15,50(\mathrm{VL})$ & $0.06(\mathrm{~L})$ & $0.27(\mathrm{VH})$ & $0.81(\mathrm{VL})$ & 1411.58 & Very low \\
\hline
\end{tabular}


Table 6: Continuation.

\begin{tabular}{|c|c|c|c|c|c|c|c|c|c|}
\hline Order & Sub-group & SMU & $\begin{array}{c}\text { CEC } \\
(\mathrm{me} / 100 \mathrm{~g})\end{array}$ & BISA (\%) & P-total $(\%)$ & K-total (\%) & $\begin{array}{c}\text { C-organic } \\
(\%)\end{array}$ & $\begin{array}{l}\text { Productivity } \\
\left(\mathrm{kg} \mathrm{ha}^{-1}\right)\end{array}$ & $\begin{array}{c}\text { Soil } \\
\text { Fertility }\end{array}$ \\
\hline \multirow{5}{*}{ Inceptisol } & \multirow{5}{*}{$\begin{array}{c}\text { Typic } \\
\text { Dystrudept }\end{array}$} & SMU 11 & $12.87(\mathrm{~L})$ & $13.04(\mathrm{VL})$ & $0.37(\mathrm{VH})$ & $0.28(\mathrm{VH})$ & $1.10(\mathrm{~L})$ & 688.73 & Very low \\
\hline & & SMU 13 & $13.85(\mathrm{~L})$ & $26.67(\mathrm{~L})$ & $0.11(\mathrm{VH})$ & $0.14(\mathrm{H})$ & $1.56(\mathrm{~L})$ & 902.36 & Low \\
\hline & & SMU 14 & $13.21(\mathrm{~L})$ & $28.98(\mathrm{~L})$ & $0.10(\mathrm{H})$ & $0.17(\mathrm{H})$ & $1.25(\mathrm{~L})$ & 797.89 & Low \\
\hline & & SMU 16 & $9.50(\mathrm{~L})$ & $14.45(\mathrm{VL})$ & $0.11(\mathrm{VH})$ & $0.16(\mathrm{H})$ & $0.80(\mathrm{VL})$ & 1201.40 & Very low \\
\hline & & SMU 18 & $10.55(\mathrm{~L})$ & $22.21(\mathrm{~L})$ & $0.09(\mathrm{H})$ & $0.17(\mathrm{H})$ & $1.10(\mathrm{~L})$ & 1089.22 & Low \\
\hline
\end{tabular}

Note: Criteria for Cation Exchange Capacity $/ \mathrm{CEC}$ (very low $/ \mathrm{VL}<5 \mathrm{me} / 100 \mathrm{~g} ;$ low $/ \mathrm{L}=5-16 \mathrm{me} / 100 \mathrm{~g} ;$ moderate $/ \mathrm{M}=17-24 \mathrm{me} / 100 \mathrm{~g} ; \mathrm{high} / \mathrm{H}=25-40$ me/100 g; very high/VH > 40 me/100 g); Base Saturation/BS (very low $/ \mathrm{VL}<20 \%$; low $/ \mathrm{L}=20-40 \%$; moderate $/ \mathrm{M}=41-60 \%$; high $/ \mathrm{H}=61-80 \%$; very high/VH> 80\%); C-organic (very low/VL<1\%; low/L= 1-2\%; moderate/M= 2.01-3.00\%; high/H=3.01-5.00\%; very high/VH $>5 \%$ ); $\mathrm{P}$-total (very low/ $\mathrm{VL}<0.03 \%$; low $/ \mathrm{L}=0.03-0.06 \%$; moderate/M=0.06-0.079\%; high $/ \mathrm{H}=0.08-0.10 \%$; very high $/ \mathrm{VH}>0.10 \%)$, K-total (very low $/ \mathrm{VL}<0.03 \%$; low $/ \mathrm{L}=$ $0.03-0.06 \%$; moderate $/ \mathrm{M}=0.07-0.11 \%$; high $/ \mathrm{H}=0.12-0.20 \%$; very high $/ \mathrm{VH}>0.20 \%$ ) (Soil Research Institute 2009); Soil fertility quality (very low = $\mathrm{VL}$; low $=\mathrm{L}$ ) adopted from Dierolf,Fairhurst and Mutert (2001).

Table 7: Multiple linear regression analysis of the CEC, BS, P-total, K-total, C-organic on the yield characteristics for Coffee robusta in Silima Pungga-Pungga sub-District, Dairi District, North Sumatra Province, Indonesia.

\begin{tabular}{|c|c|c|c|c|c|c|c|}
\hline Yield Characteristics & Model & Sum of Squares & df & Mean Square & $\mathrm{F}$ & Sig. & $r^{2}$ \\
\hline \multirow{3}{*}{ Productivity } & Regression & 629804.71 & 5 & 125960.94 & $6.68^{*}$ & 0.045 & $89.30 \%$ \\
\hline & Residual & 75448.93 & 4 & 18862.23 & & & \\
\hline & Total & 705253.64 & 9 & & & & \\
\hline \multirow{3}{*}{100 grains of dry weight } & Regression & 108.75 & 5 & 21.75 & $2.58^{\mathrm{ns}}$ & 0.189 & $76.40 \%$ \\
\hline & Residual & 33.67 & 4 & 8.42 & & & \\
\hline & Total & 142.42 & 9 & & & & \\
\hline
\end{tabular}

Note: *significant at the level of $95 \%$; ns= not significant at the level of $95 \%$.

\section{DISCUSSION}

The overlay result showed ten of 18 SMUs were selected for Coffee robusta in Silima Pungga-Pungga sub-District, Dairi District, North Sumatra Province and dominant at an altitude ranged of 400 to $600 \mathrm{~m}$ above sea level (MASL) covering an area of 1,069.19 ha compared to an altitude of 600 to $800 \mathrm{MASL}$ at an area of $810.92 \mathrm{ha}$. According to Directorate General of Estate Crops (2015) the appropriate altitude for growing the Coffee robusta ranged from 100 until 600 MASL and the highly suitable land suitability class at an altitude of 300 to 500 MASL and a slope of 0 to $8 \%$. Arvi, Syakur and Karim (2019) stated that an altitude and slope did not significant and negatively correlate $\mathrm{d}(\mathrm{R}=-0.372$ and -0.182$)$ on the weight of the net beans for Coffee arabica Gayo, it means that at each increase in altitude 200 MASL and the slope of 1\% could be decreased the net beans weight of $0.14 \%$ and $0.007 \%$ per hectare, respectively. The higher an altitude causes the lower temperature will result that the ripening process of the fruit the slower and lower the fruit filling. Salima, Karim and Sugianto (2012) reported that the flat slope (0 until 8\%) could be produced a higher yield for Coffee arabica. The slope relating to the availability of C-organic, Al-dd, pH, P-available, and N-total.
Based o $\mathrm{n}$ ten $\mathrm{S}$ MUs selected, found in two representative soil profiles such as profile 1 (umbric, cambic, incepti s ol, u d ept, dystrudept, typic dystrudept, and the horizon Ap-B-C), and the profile 10 (ochric, it does not have a characteristic subsurface horizon, entisol, orthent, udorthent, typic $\mathrm{ud}$ orth e $\mathrm{nt}$, and the horizon Ap-A-C). The result indicated that the order inceptisol has the characteristics of CEC, C-organic, and BS were higher ranged of 13.21 to 21.09 me/100 g; 0.49 to $1.70 \%$; and 15.13 to $18.51 \%$, respectively compared to entisol (Table 5). According to Schaetzl, Krist Junior and Miller (2012) the index soil productivity (ISP) for inceptisol had a value of 9 and was higher compared to entisol of 6. Hadi, Sutikto and Bowo (2019) reported that the clas sification of the inceptisol order, udept sub-order, dystrud e pt great group, typic dystrudept sub-group from Coffee robus ta plantations in Rayap-2 locations, Jember District had ISP value at 7 and was higher compared to the entisol order, orthent sub-order, udorthent great group, typic udorthent sub-group with ISP value of 6 in Sidomulyo location, Jember District. The productivity index based on soil taxonomy has a very strong correlation $(\mathrm{r}=0.84)$ with the productivity for Coffee robusta. The higher the ISP value will result the higher the productivity for Coffee robusta. 
Based on the physical properties of ten SMUs selected, showed that the consistency, structure, and texture of soil on both so il orders were classified as supporting root activity in elon gation and growth of the roots for Coffee robusta. According to Khalil et al. (2015) the soil characteristics including the physical, chemical, and biological caused the chain changes in physiological, biological, and chemical on the growth, yield, and quality of biomass, and fiber plants. Pardo, Amato and Chiarandà (2000) stated that the soil structure could be supporting the growth of Cicer arietinum and the spread of roots to absorb water and nutrients. Avelino et al. (2002) reported that soil texture and acidity could affect the quality of coffee beans. Gil et al. (2012) reported that soil texture could affect the movement and availability of water, air, and nutrients. Chaudhari et al. (2013) reported that soil texture could affect the bulk density of soil and stimulate the yield of plants. Vetterlein et al. (2007); Bada and Raji (2010) also reported that soil texture was significant for the absorption efficiency of plants.

Inceptisol order has greater the soil chemical properties compared to entisol from ten SMUs selected for Coffee robusta in Silima Pungga-Pungga sub-District, Dairi District. It is due to the highest content of CEC, BS, and C-organic found in the inceptisol order. The $\mathrm{C}$-organic content could be affected by cation exchange with the result that nutrients become available to support the growth and yield for Coffee robusta. In addition, on the Ap horizon with the soil depth of 0-34 cm in the inceptisol order obtained the ratios of $\mathrm{Ca}^{2+} / \mathrm{K}^{+}$and $\mathrm{Mg}^{2+} / \mathrm{K}^{+}$of 5.32 and 5.53 , respectively. The result indicated the nutrients balance of $\mathrm{Ca}$ and $\mathrm{Mg}$ which can support plant roots to absorb the nutrients needed and affect to yield plant. According to Sousa et al. (2018) the soil organic matter significantly increased the content of $\mathrm{N}$-total and $\mathrm{S}$ in the leaves of coffee plants from Minas Gerais. Kufa (2011); Kilambo et al. (2015) stated that humus colloids have the higher cationic absorption compared to clay colloids with the result that the higher organic matter could be increasing the value of soil CEC. Loide (2004) stated that the relationship between $\mathrm{K} / \mathrm{Mg}$ was significantly and positive correlation $(\mathrm{r}=0.889)$ on the yield plants. The yield increased until the ratio of $\mathrm{K} / \mathrm{Mg}$ achieved $0.6-0.7$ : 1 . The potassium content increases, it occurs a decrease in the yield of Trifolium pratense, except the magnesium nutrient was added to manage the balance of $\mathrm{K} / \mathrm{Mg}$. In addition, the overage of Mg-exchangeable in the soil which is un-balanced with $\mathrm{Ca}$ will deteriorate the physiological characteristics of the roots and lead to decreased the yield plant.

The soil fertility assessment of the inceptisol in the location research was greater than the entisol. It was caused the highest content of CEC, BS, P-total, and C-organic found in the inceptisol of $14.94 \mathrm{me} / 100 \mathrm{~g} ; 28.98 \% ; 0.37 \%$, and $1.70 \%$, respectively. According to Malavolta et al. (1979); Chaves, Pavan and Miyazawa (1991) the increased Ca-exchangeable content had a linear and significant relationship with increased the yield of coffee beans. Bradl (2004) reported that organic matter had a positive correlation with soil cation exchange. Alves et al. (2015) reported that base saturation up to $60 \%$ was significantly and positively correlated $(\mathrm{R}=0.722$ and 0.9883$)$ to the dry weight of Crambe abyssinica roots at 35 and 55 days after growing.

The effect of CEC, BS, P-total, K-total, and C-organic were significantly affected on the productivity and was not significant effect on the 100 grains of dry weight for Coffee robust $a \mathrm{w}$ ith the determination coefficient by $89.30 \%$ and $76.40 \%$, respectively. According to Supriadi, Randriani and Towaha (2016) the content of C-organic, CEC, N-total, and soil $\mathrm{pH}$ significantly increased on the normal beans and 100 beans weight for Coffee arabica in the highlands of Garut District, West Java Province, Indonesia. Cyamweshi et al. (2014) stated that s o il $\mathrm{pH}$, Cacontent, $\mathrm{P}$-available, N-total, and C-organic were positively correlated with the determination coefficient by $71 \% ; 56 \% ; 62 \% ; 30 \%$, and $52.7 \%$ respectively of coffee yield in Nyamagabe District, Southern Rwanda Province. Clemente et al. (2013) stated that $\mathrm{K}$ nutrient content had the quadratic response to growth, bean size, and coffee bean yield.

\section{CONCLUSIONS}

The result obtained ten from 18 SMUs selected for Coffee robustaand had the highest area in sequentially, such as SMU 11, 14, and 1. Area growing for Coffee robusta dominant at an altitude ranged of 400 until 600 MASL with an area of 1,069.19 ha. Based on the ten SMUs selected, found in two representative soil profiles, include the profile 1 (SMU 1, 2, 8, $9,11,13,14,16,18)$ covering an area of $1,703.30$ ha with the inceptisol, had horizon of Ap-B-C, soil structure of granular until blocky, soil consistency of the soft until slightly hard, the soil texture of the sandy clay loam until clay, and bulk density ranged of 1.04 to $1.12 \mathrm{~g} \mathrm{~cm}^{-3}$, the chemical properties was classified as very low until high,and soil fertility was classified as very low. In profile 10 (SMU 10) covering an area of 176.81 ha with the entisol, had horizon of Ap-A-C, soil structure of the granular, soil consistency of the soft until very soft, soil texture of sandy loam until sandy clay loam, bulk density ranged of 1.18 to $1.26 \mathrm{~g} \mathrm{~cm}^{-3}$, the chemical properties was classified as very low until low, and the soil fertility was classified as very low. Effect of CEC, BS, P-total, K-total, and $\mathrm{C}$-organic significantly increased the productivity Coffee robusta by $89.30 \%$. However, the effect was not significant to the 100 grains of dry weight.

\section{ACKNOWLEDGMENTS}

This research was supported by Ministry of Research, Technology, and Higher Education, Republic Indonesia for providing financial assistance through by dissertation grant program. 


\section{REFERENCES}

ALVES, J. M. et al. Effect of base saturation and nitrogen dose on cultivation of crambe. African Journal of Agricultural Research, 10(1):14-22, 2015.

ARVI, D.; SYAKUR, S.; KARIM, A. The elevation relation and slope toward Gayo 1 arabica coffee (Coffea arabica) production in Gayo Lues. Jurnal Ilmiah Mahasiswa Pertanian,4(4):596-602, 2019.

AVELINO, J. et al. Identifying terroir coffees in Honduras. Plantations, Recherche, Développement, 6-16p, 2002.

BADA, B. S.; RAJI, K. A. Phytoremediation potential of kenaf (Hibiscus cannabinus L.) grown in different soil textures and cadmium concentrations. African Journal of Environmental Science and Technology, 4(5):250$255,2010$.

BRADL, H. B. Adsorption of heavy metal ions on soils and soils constituents. Journal of Colloid and Interface Science, 277(1):1-18, 2004.

CHAUDHARI, P. R. et al. Soil bulk density as related to soil texture, organic matter content and available total nutrients of Coimbatore soil. International Journal of Scientific and Research Publications, 3(2):1-8, 2013.

CHAVES, J. C. D.; PAVAN, M. A.; MIYAZAWA, M. Chemical speciation of soil solution to assess calcium and aluminum uptake by coffee roots. Pesquisa Agropecuaria Brasileira, 26(3):447-453, 1991.

CLEMENTE, J. M. et al. Effect of $\mathrm{N}$ and $\mathrm{K}$ doses in nutritive solution on growth, production and coffee bean size. Revista Ceres, 60(2):279-285, 2013.

CYAMWESHI, R. A. et al. Enhancing nutrient availability and coffee yield on acid soils of the central plateau of Southern Rwanda. Global Journal of Agricultural Research, 2(2):44-55, 2014.

DIEROLF, T. S.; FAIRHURST, T. H.; MUTERT, E. W. Soil fertility kit. a toolkit for acid, upland soil fertility management in Southeast Asia. Watten Estate Road, Singapore: PPI. 2001. 149p.

DIRECTORATE GENERAL OF ESTATE CROPS. Tree crop estate statistics of Indonesia 2014-2016, Coffee. Jakarta, Indonesia: Ministry of Agriculture, 2015. 97p.

GIL, P. M. et al. Effect of soil water-to-air ratio on biomass and mineral nutrition of avocado trees. Journal of Soil Science and Plant Nutrition, 12(3):609-630, 2012.
HADI, R. M. P.; SUTIKTO, T.; BOWO, C. Soil productivity index based on soil taxonomy and its correlation with coffee productivity in several soil orders. Jurnal Tanah dan Lingkungan, 21(1):7-12, 2019.

INDONESIAN AGENCY FOR AGRICULTURAL RESEARCHAND DEVELOPMENT. Coffee cultivation technology, application in smallholder plantations. Jakarta, Indonesia: IAARS Press, 2015. 124p.

KHALIL, H. P. S. A. et al. The role of soil properties and it's interaction towards quality plant fiber: A review. Renewable and Sustainable Energy Reviews, 43:10061015, 2015.

KILAMBO, D. L. et al. Effect of soils properties on the quality of compact arabica hybrids in Tanzania. American Journal of Research Communication, 3(1):15-19, 2015.

KUFA, T. Chemical properties of wild coffee forest soils in Ethiopia and management implications. Agricultural Sciences, 2(4):443-450, 2011.

LOIDE, V. About the effect of the contents and ratios of soil's available calcium, potassium and magnesium in liming of acid soils. Agronomy Research, 2(1):71-82, 2004.

MALAVOLTA, E. et al. Calcium problems in Latin America. Communications in Soil Science and Plant Analysis, 10(1-2):29-40, 1979.

MARBUN, P. et al. Classification of inceptisol soil on robusta coffee plantation in silima pungga-pungga district. International Journal of Sciences Basic and Applied Research, 30(5):20-27, 2016.

MARBUN, P. et al. Classification of andisol soil on robusta coffee plantation in Silima Pungga-Pungga District. IOP Conference Series: Earth and Environmental Science, 122:e012045, 2018.

PARDO, A.; AMATO, M.; CHIARANDÀ, F. Q. Relationships between soil structure, root distribution and water uptake of chickpea (Cicer arietinum L.). Plant growth and water distribution. European Journal of Agronomy, 13(1):39-45, 2000.

SALIMA, R.; KARIM, A.; SUGIANTO, S. Evaluation criteria of land suitability to arabica Gayo 2 coffee in the gayo highlands. Jurnal Manajemen Sumberdaya Lahan, 1(2):194-206, 2012.

SCHAETZL, R. J.; KRIST JUNIOR, F. J.; MILLER, B. A. A taxonomically based ordinal estimate of soil productivity for landscape-scale analyses. Soil Science, 177(4):288299, 2012. 


\section{SOIL RESEARCH INSTITUTE. Technical guide 2:}

Chemical analysis of soil, plants, water and fertilizer.

Bogor, Indonesia: Ministry of Agriculture, 2009. 246p.

SOUSA, J. S. et al. Relationship between coffee leaf analysis and soil chemical analysis. Revista Brasileira de Ciência do Solo, 42:1-13, 2018.

\section{STATISTICS OF INDONESIA. Coffee exports by main} destination countries, 2000-2019. Jakarta, Indonesia: Statistics of Indonesia, 2019. Available in: $<$ https:// www.bps.go.id/statictable/2014/09/08/1014/ekspor-kopimenurut-negara-tujuan-utama-2000-2019.html>. Access in: June, 17, 2020.
STATISTICS OF SUMATERA UTARA. Sumatera utara province in figures 2018. Medan, Indonesia: Statistics of Sumatera Utara, 2018. 762p.

SUPRIADI, H.; RANDRIANI, E.; TOWAHA, J. Correlation between altitude, soil chemical properties, and physical quality of arabica coffee beans in highland areas of Garut. Jurnal Tanaman Industri dan Penyegar, 3(1):45-52, 2016.

VETTERLEIN, D. et al. Impact of soil texture on temporal and spatial development of osmotic-potential gradients between bulk soil and rhizosphere. Journal of Plant Nutrition and Soil Science, 170(3):347-356, 2007. 